\title{
The influence of Mn redox cycling on soil C stability
}

\section{ELIZABETH L PAULUS AND PETER VITOUSEK}

\author{
Stanford University
}

Presenting Author: paulus@stanford.edu

Soil organic matter (SOM) is the largest reservoir of potentially dynamic carbon (C) on Earth, with more than three times the quantity as in the atmosphere. Even a small tip of the balance between SOM formation and SOM decomposition can have disproportionate effects on the concentration of $\mathrm{CO}_{2}$ in the atmosphere, and so on climate. Most $\mathrm{C}$ in SOM is in pools that turn over slowly, due to chemical recalcitrance and/or physical protection. Evidence suggests that manganese (Mn) can enhance SOM decomposition, including pools thought to degrade slowly. This influence depends on the metal's abundance and oxidation state. When soil redox conditions fluctuate, Mn cycles through $\mathrm{Mn}(\mathrm{II}), \mathrm{Mn}(\mathrm{III})$, and $\mathrm{Mn}(\mathrm{IV})$. Microbes can couple Mn reduction and oxidation across oxic-anoxic interfaces in soil pores, generating $\mathrm{Mn}$ species that can degrade SOM and release C. Studies on the relationship between $\mathrm{Mn}$ and soil $\mathrm{C}$ stability have investigated $\mathrm{Mn}$ in forest litter. It remains unclear what environmental conditions potentiate the tight coupling of $\mathrm{Mn}$ cycling and POM oxidation and if the same relationship between $\mathrm{Mn}$ and litter degradation observed in forests exists in other ecosystems.

We address these questions using the Kohala climate gradient on the Island of Hawai' $\mathrm{i}(<300 \mathrm{~mm} / \mathrm{yr}$ precipitation to $>3300$ $\mathrm{mm} / \mathrm{yr}$ ). We hypothesize that climate is the dominant control of $\mathrm{Mn}$ oxidation state and availability, and so of Mn-cycling microbial community compositions and activities on the Kohala gradient. To test this hypothesis, we analyze how Mn availability, Mn-cycling microbial gene abundance and activity, and soil $\mathrm{C}$ abundance and composition change with climate.

Preliminary data indicate that plant-available (reduced) $\mathrm{Mn}$ correlates with soil C abundance. Further research on Mn-cycling microbial community compositions and activities should clarify the mechanisms at play. 